\title{
BRINKMAN - FORCHHEIMER - DARCY FLOW PAST AN IMPERMEABLE SPHERE EMBEDDED IN A POROUS MEDIUM
}

\author{
Gheorghe Juncu
}

\begin{abstract}
The flow past an impermeable sphere embedded in a fluid saturated porous medium was studied numerically considering valid the Brinkman-Forchheimer-Darcy (or Brinkman-Hazen-Dupuit-Darcy) model. The flow is viscous, laminar, axisymmetric, steady and incompressible. The porous medium is isotropic, rigid and homogeneous. The stream function - vorticity equations were solved numerically in spherical coordinates system by a multigrid method. The influence of the Darcy number and Forchheimer term on the velocities field was investigated for two boundary conditions on the surface of the sphere: slip and no - slip.
\end{abstract}

\section{Introduction}

Flow in a fluid saturated porous medium has broad applications in many environmental, industrial and life science processes. The mathematical models for flow in fluid saturated porous media are well discussed and presented by Bear \& Bachmat [1], Kaviany [8] , Lage [9] and Nield \& Bejan [11]. An exhaustive presentation of these models is outside the aims of the present work.

Key Words: saturated porous medium, sphere, axisymmetric flow, stream function, vorticity, no slip boundary condition, slip boundary condition.

2010 Mathematics Subject Classification: Primary 76S05

Received: October, 2014.

Revised: January, 2015.

Accepted: February, 2015. 
Many hundreds of papers deal with flow in fluid saturated porous medium but only few considered flow around solid inclusions embedded in a porous medium. Analytical solutions for the Darcy flow past an impervious sphere embedded in a fluid saturated porous medium can be viewed in Romero [13] and Feng \& Michaelides [6]. In these articles, the slip condition is satisfied on the surface of the sphere. The incompressible steady viscous flow past a sphere embedded in a constant porosity medium using the Brinkman model with no slip boundary condition on the surface of the sphere was analysed by Brinkman [4], Berman [2], Pop \& Ingham [12], Ganapathy [7], Charya \& Murthy [5] and Wang [15]. In these articles, an analytical exact solution for the stream function was obtained for the case when the velocity far away from the sphere is uniform. The axisymmetric steady incompressible flow past an impermeable sphere embedded in a porous medium using the Brinkman model with a uniform shear instead of a uniform velocity far away from the sphere and no slip boundary condition was investigated by Rudraiah et al. [14].

An analytic solution for the problem of the incompressible steady viscous flow past an impermeable cylinder / sphere embedded in a porous medium using the Brinkman model with Navier boundary condition on the surface of the sphere was obtained by Leont'ev [10]. Leont'ev [10] considers that setting the no - slip condition when using the seepage equations with higher spatial derivatives (Brinkman, Darcy Lapwood Brinkman and other models) is generally inadequate.

Thus, for the flow past an impervious sphere embedded in a fluid saturated porous medium only the Darcy and Brinkman models were used. The aim of this work is the numerical analysis of the flow past an impermeable sphere embedded in a fluid saturated porous medium using the Brinkman Forchheimer - Darcy model. This problem was not investigated until now. The present computations are focused on the influence of the Darcy number and Forchheimer term on the velocities field for different values of the sphere Reynolds number and two boundary conditions on the surface of the sphere: slip and no - slip.

This paper is organized as follows. In Sect. 2 we describe the mathematical model of the problem. Section 3 presents the numerical algorithm. The numerical experiments made and the results obtained are presented in Sect. 4. Finally, some concluding remarks are briefly mentioned in Sect. 5 .

\section{Model Equations}

Consider the laminar, viscous, steady, axisymmetric, incompressible flow of a Newtonian fluid with a superficial velocity $U_{0}$ past an impervious sphere embedded in a porous medium with permeability $K$. The porous medium is 
rigid, isotropic, homogeneous and fluid saturated. The following additional assumptions are considered valid:

- during the flow, the volume and shape of the sphere are constant;

- surface tension effects are considered negligible;

- the physical properties of the sphere and ambient porous medium are uniform, isotropic and constant;

- no phase change.

Under these assumptions, the dimensionless Brinkman - Forchheimer - Darcy model equations (the radius of the sphere, $a$, is considered as the length scale and the free stream velocity, $U_{0}$, as the velocity scale) are:

- continuity equation

$$
\nabla \cdot V=0
$$

- momentum equation

$$
-\nabla p+\frac{2}{R e} \nabla^{2} V-\frac{\varepsilon}{2 R e D a} V-\frac{\varepsilon C_{F}}{2 D a^{1 / 2}}\|V\| V=0
$$

where $V$ is the Darcy dimensionless velocity vector $V=\left(V_{R}, V_{\theta}\right), p$ is the dimensionless local average pressure, $\varepsilon$ is the porosity of the porous medium, $C_{F}$ the Forchheimer constant and

$$
D a=\frac{K}{d^{2}}, R e=\frac{U_{0} d \rho}{\mu},\|V\|=\sqrt{V_{R}^{2}+V_{\theta}^{2}} .
$$

In the previous relations $d$ is the diameter of the sphere, $d=2 a, \mu$ is the dynamic viscosity of the fluid and $\rho$ is the density of the fluid. Also, the Darcy and sphere Reynolds numbers are symbolized by $D a$ and $R e$.

For axisymmetric flow, the dimensionless vorticity vector

$$
\zeta=\nabla \times V
$$

has only one non-zero component, i.e.

$$
\zeta=\frac{\partial V_{\theta}}{\partial r}-\frac{1}{r} \frac{\partial V_{R}}{\partial \theta}+\frac{V_{\theta}}{r}
$$

Defining the dimensionless stream function by,

$$
V_{R}=-\frac{1}{r^{2} \sin \theta} \frac{\partial \psi}{\partial \theta}, V_{\theta}=\frac{1}{r \sin \theta} \frac{\partial \psi}{\partial r}
$$


and eliminating $V_{R}$ and $V_{\theta}$ from (3) and (4), we obtain the stream function equation in dimensionless spherical coordinate system $(r, \theta)$ as,

$$
\frac{\partial^{2} \psi}{\partial r^{2}}+\frac{\sin \theta}{r^{2}} \frac{\partial}{\partial \theta}\left(\frac{1}{r \sin \theta} \frac{\partial \psi}{\partial r}\right)=\zeta r \sin \theta
$$

Using the relation

$$
\nabla^{2} V=\nabla \nabla \cdot V-\nabla \times(\nabla \times V)
$$

the momentum equation (2) can be rewritten as,

$$
-\nabla p+\frac{2}{R e} \nabla \times \zeta-\frac{\varepsilon}{2 R e D a} V-\frac{\varepsilon C_{F}}{2 D a^{1 / 2}}\|V\| V=0
$$

Applying the curl operator to equation (6) and taking into consideration that

$$
\nabla \times \nabla p=0, \nabla \times(f V)=f \nabla \times V+\nabla f \times V,
$$

for any scalar function $f$, it results, in dimensionless spherical coordinate system $(r, \theta)$,

$\frac{\partial^{2} \zeta}{\partial r^{2}}+2 \frac{\partial \zeta}{\partial r}+\frac{1}{r^{2}}\left(\frac{\partial^{2} \zeta}{\partial \theta^{2}}+\cot \theta \frac{\partial \zeta}{\partial \theta}-\frac{\zeta}{\sin ^{2} \theta}\right)-\frac{\varepsilon}{4 D a} \zeta-\frac{\varepsilon C_{F} R e}{4 D a^{1 / 2}}(\|V\| \zeta+\nabla\|V\| \times V)=0$

The boundary conditions are:

- axis of symmetry, $\theta=0, \pi$,

$$
\psi=\zeta=0
$$

- surface of the sphere, $r=1$,

(i) no - slip

$$
\psi=\frac{\partial \psi}{\partial r}=0
$$

(ii) slip

$$
\psi=0, \zeta=\frac{1}{\sin \theta} \frac{1+2 \beta}{\beta} \frac{\partial \psi}{\partial r}
$$

- free stream, $r \rightarrow \infty$,

$$
\frac{\partial}{\partial r}\left(\psi-\frac{1}{2} r^{2} \sin ^{2} \theta\right)=0, \zeta=0 .
$$

where $\beta$ is the dimensionless slip coefficient, [10] ( $\beta=0$ means no slip while $\beta \rightarrow \infty$ means perfect slip). A value of $\beta$ between these two limits corresponds to partial slip on the surface of the sphere. The present mathematical model is composed by equations (5), (7) and boundary conditions (8),(9),(10),(11). 


\section{Method of Solution}

The mathematical model equations were solved numerically. The radial coordinate $r$ was replaced by $x$ using the transformation $r=\exp (x)$. As a result, the use of a constant discretization parameter for $x$ made it possible to obtain a more dense mesh near the surface of the sphere where the gradients are large and where an accurate numerical approximation is needed. The central finite difference scheme was used to discretize the equations of the mathematical model.

The algorithm employed to solve the discrete equations is the classical multigrid (MG) - Full Approximation Storage (FAS) algorithm, [3], suitable for both linear and nonlinear problems. The structure of the MG cycle is: 1) cycle of type $\mathrm{V} ; 2$ ) two smoothing steps are performed before the coarse grid correction and one after; 3 ) prolongation by bilinear interpolation for corrections; 4) restriction of residuals by full weighting. Two smoothing algorithms were tested: point Gauss Seidel (PGS) and alternating line Gauss - Seidel (ALGS). The spatial discretization steps on the coarsest grid are, $\Delta \theta=\pi / 32, \Delta x=1 / 32$. Numerical experiments were made on fine meshes with the discretization steps, $\Delta \theta=\pi / 128, \Delta x=1 / 128$, $\Delta \theta=\pi / 256, \Delta x=1 / 256$, and $\Delta \theta=\pi / 512, \Delta x=1 / 512$. The error criterion employed is: the discrete $L_{2}$ norm of the residuals is smaller than $10^{-8}$. It must be also mentioned that the numerical algorithm converged for all parameters values employed in the numerical experiments made.

\section{Results}

The dimensionless parameters of the Brinkman - Forchheimer - Darcy model are: $C_{F}, D a, R e$ and $\varepsilon$. The numerical results presented in this section were obtained for numerical values of the Re number in the range $R e \leq 10$.

The value considered in this work for $\varepsilon$ is, $\varepsilon=0.9$. This selection does not restrict the area of interest of the present results. The Darcy number, $D a$, takes values in the range, $D a \leq 10$. The value considered for the Forchheimer constant is, $C_{F}=0.55$. The numerical experiments made focus on the following problem: the influence of the Darcy number and Forchheimer term on the flow characteristics for the no slip or slip wall boundary condition. The nu-

merical results obtained for each boundary condition are presented separately. The quantities used to analyse the present computations are the vorticity on the surface of the sphere and the velocity profiles. 


\subsection{No-slip boundary condition}

From the beginning it must be mentioned that the solutions of the Brinkman - Forchheimer - Darcy model have all the characteristics of the creeping flow solutions (symmetric $\zeta_{s}$, symmetric / antisymmetric velocities, and so on) (the axis of symmetry / antisymmetry is $\theta=\pi / 2$ ). Under these conditions, the influence of the Forchheimer term on the flow field will be investigated comparing the present numerical results with the analytical solution of Wang [15]. The analytical solution of Wang [15] was derived considering $C_{F}=0$.

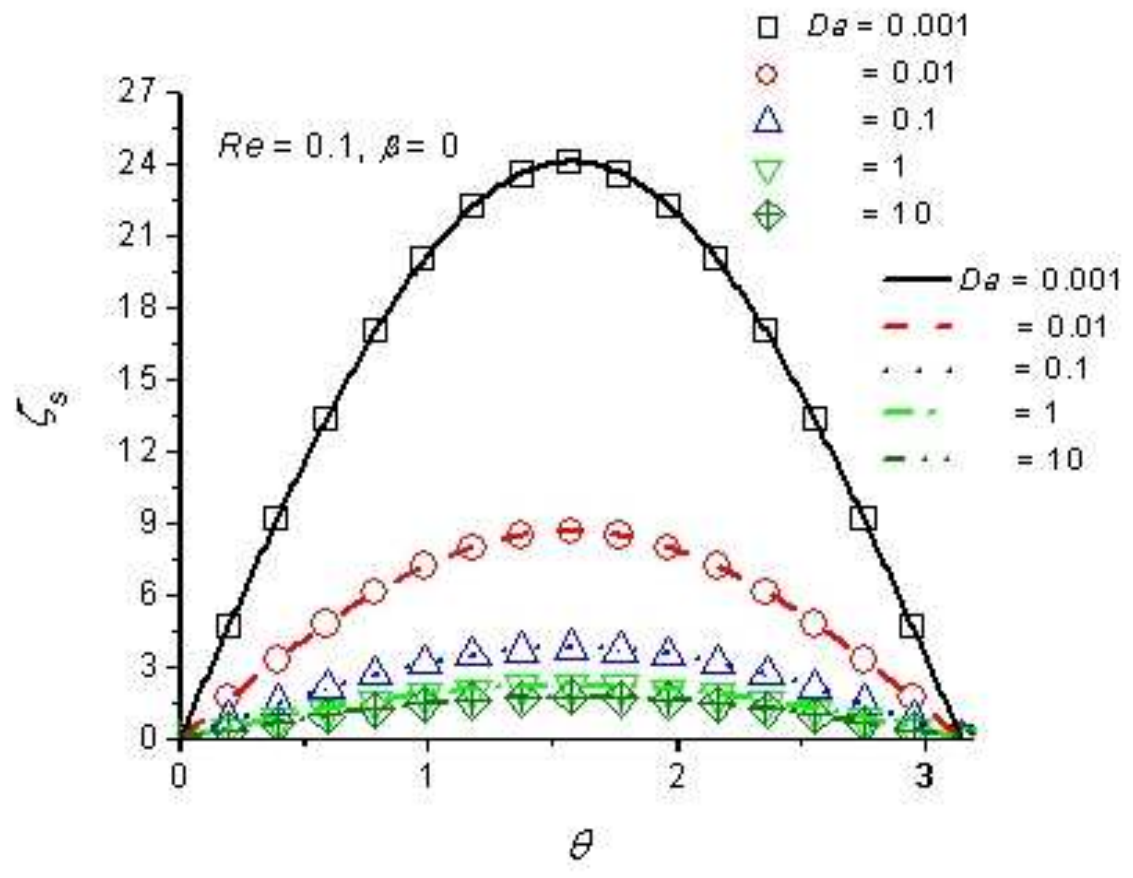

Figure 1: The influence of the $D a$ number on the surface vorticity for $R e=$ $0.1, \beta=0$ (no - slip boundary condition) and $\varepsilon=0.9$.

Figures 1 to 3 shows the influence of the Da number on the surface vorticity, $\zeta_{s}$, for $R e=0.1,1,10$. The symbols from figures 1 to 3 refer to the analytical solution of Wang [15]. The present numerical results are depicted as continuous lines. The numerical data presented in figures 1 to 3 shows that:

- for a given $R e$ number, the decrease in $D a$ increases the values of the surface vorticity; 


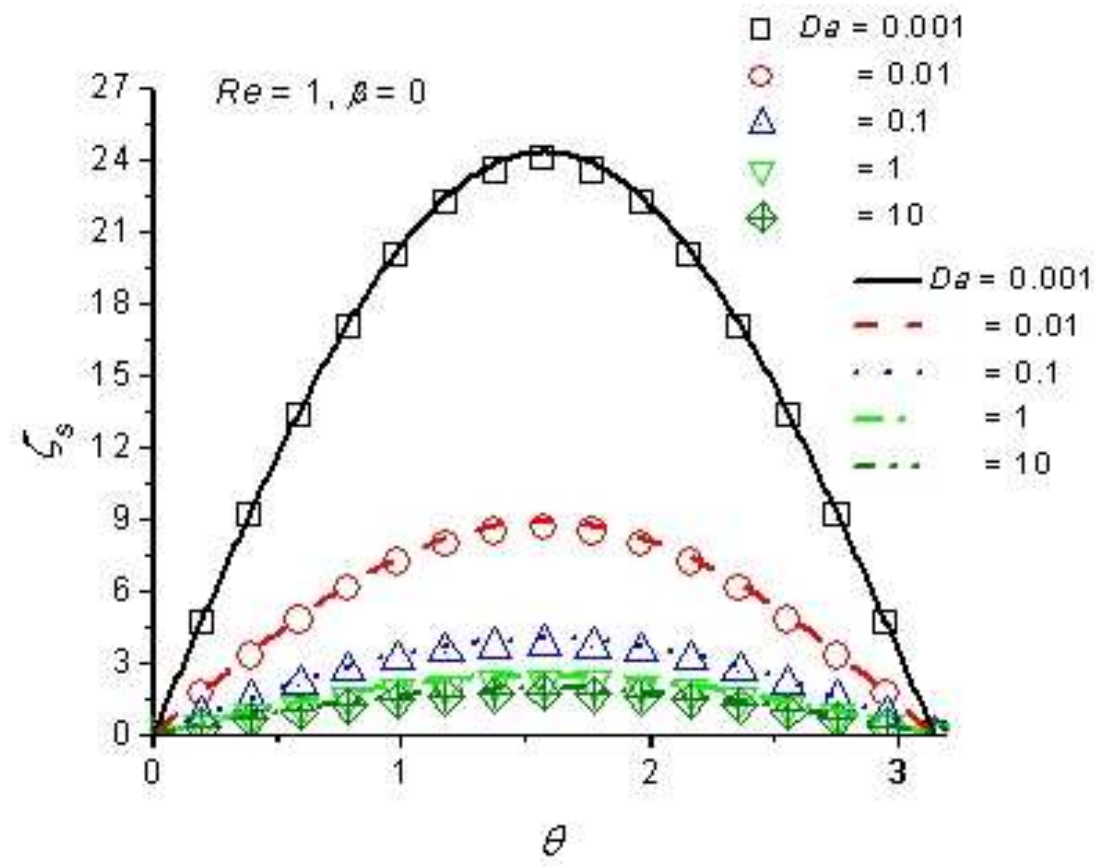

Figure 2: The influence of the $D a$ number on the surface vorticity for $R e=1$, $\beta=0$ (no - slip boundary condition) and $\varepsilon=0.9$.

- the influence of the Forchheimer term increases with the increase in Re; for $R e=0.1,1$, the agreement between the present numerical solutions and the analytical solution of Wang [15] is very good;

- for $R e=10$, the differences between the present numerical solutions and the analytical solution of Wang [15] can not be considered negligible; the decrease in $D a$ amplifies these differences;

- for $R e>1$ and a given $D a$ number, the increase in $R e$ increases the values of the surface vorticity.

The velocity profiles provide additional information concerning the influence of the Darcy and Forchheimer terms on the flow field. From the numerical experiments made, the velocity profiles computed for $D a=0.001$ and $R e=$ 1,10 , were selected (figures 4 and 5 ). The symbols depicted in figures 4 and 5 were calculated with the analytical solution from [15]. 


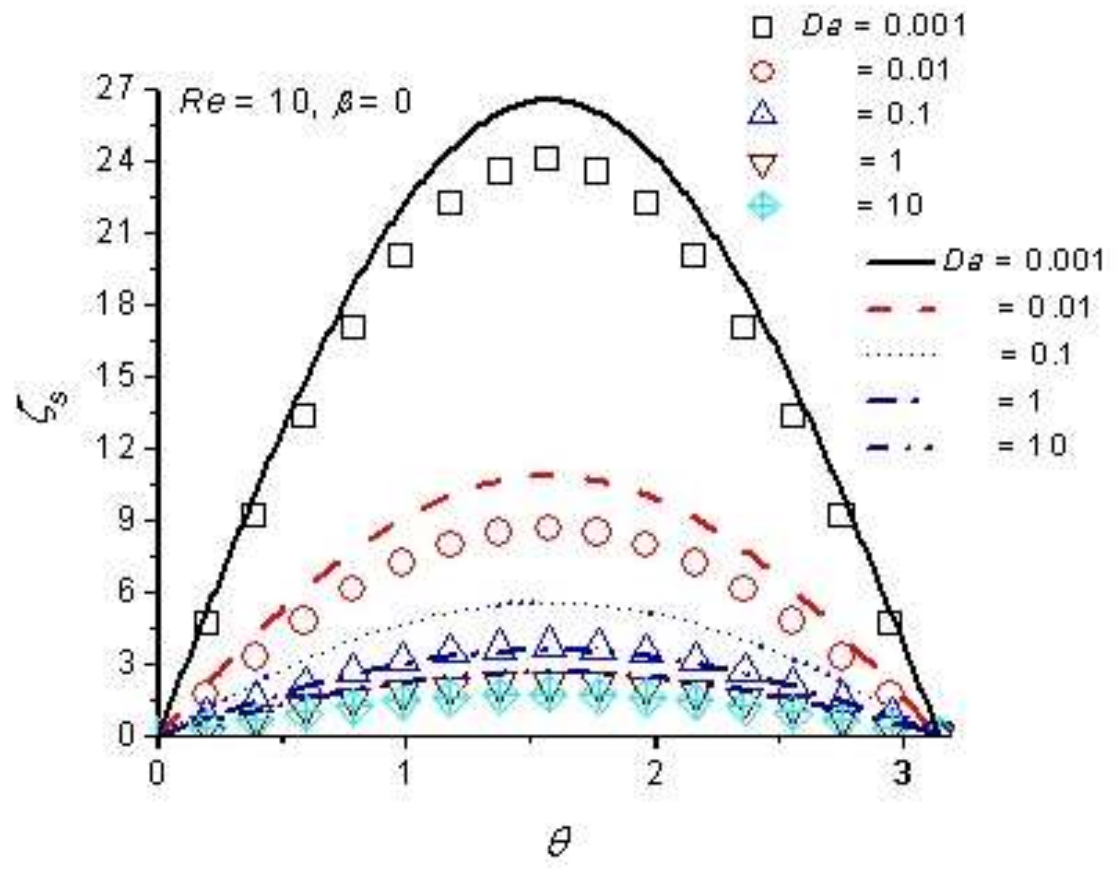

Figure 3: The influence of the $D a$ number on the surface vorticity for $R e=$ $10, \beta=0$ (no - slip boundary condition) and $\varepsilon=0.9$.

From the numerical experiments made and the results presented in figures 4 and 5 , the following observations can be made:

- the velocities vary from zero at the surface of the sphere to the asymptotic values of the unperturbed flow with the increase in $r$; the variation of the tangential velocity is not monotonic for all situations; in some cases, especially for small values of the $D a$ number, there is a velocity overshoot near the surface of the sphere (the tangential velocity has characteristic ears);

- the influence of the $D a$ number on the velocity profiles is significant only inside a viscous film that develops on the surface of the sphere; the decrease in $D a$ decreases the thickness of the viscous film;

- the Forchheimer term increases the gradient of the tangential velocity near the surface of the sphere; this increase becomes significant for Re $>$ 


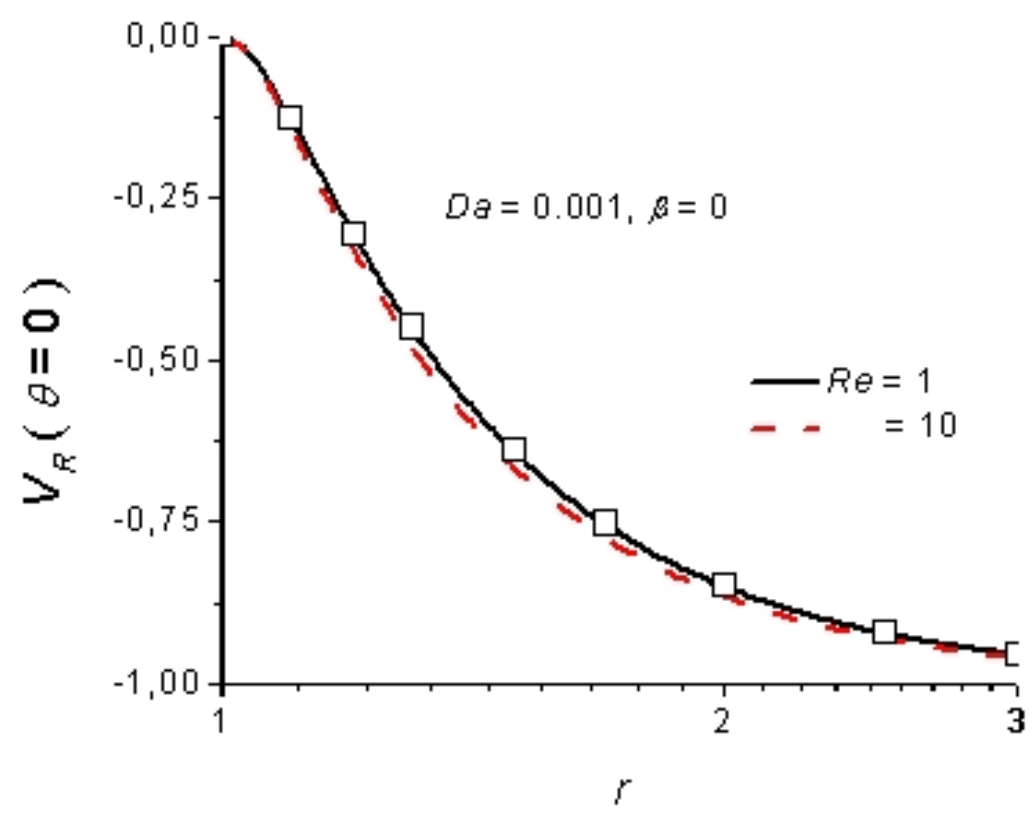

Figure 4: Radial velocity profiles for $\beta=0$ (no-slip boundary condition), $D a=0.001, R e=1,10$ and $\varepsilon=0.9$.

1; the effect of the Forchheimer term on the radial velocity in the vicinity of the surface of the sphere is negligible.

Thus, from the results presented in this section, the following conclusion can be drawn:

- the effect of the Forchheimer term on the velocity profiles is significant only for $R e>1$; the decrease in $D a$ amplifies this effect; for $R e \leq 1$, the analytical solution of Wang [15] can be used to calculate the velocities field.

\subsection{Slip boundary condition}

The numerical results presented in this section were calculated for $\beta=1$. As for the case $\beta=0$ (i.e. no - slip boundary condition), it must be mentioned that the solutions of the Brinkman - Forchheimer - Darcy model have the 


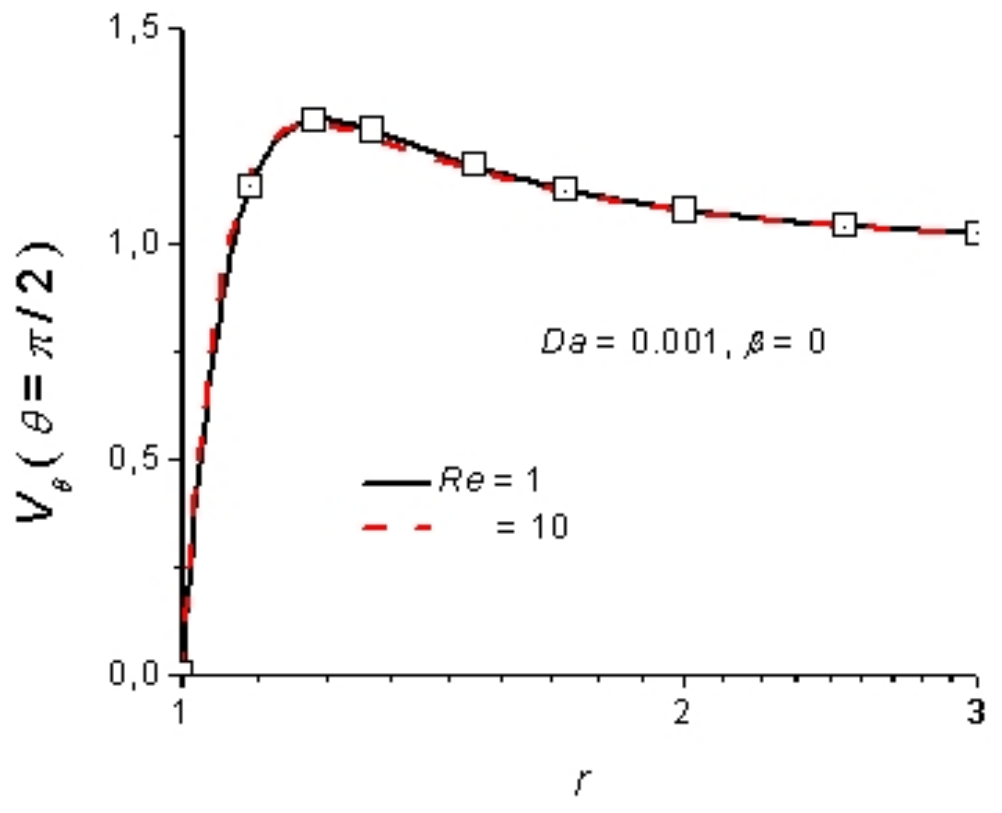

Figure 5: Tangential velocity profiles for $\beta=0$ (no-slip boundary condition), $D a=0.001, R e=1,10$ and $\varepsilon=0.9$.

characteristics of the creeping flow solutions (symmetric $\zeta_{s}$, symmetric / antisymmetric velocities, and so on) (the axis of symmetry / antisymmetry is $\theta=\pi / 2)$. The comparison criterion employed for slip boundary condition is the analytical solutions from [10]. The analytical solution of Leont'ev [10] was obtained for the case $C_{F}=0$.

The influence of the $D a$ number on the surface vorticity, $\zeta \mathrm{s}$, for $R e=$ $0.1,1,10$ can be viewed in figures $6,7,8$. The velocity profiles computed for $D a=0.001,1$ and $R e=10$ are plotted in figures 9 and 10 . The symbols depicted in figures 6-10 represent the results provided by the analytical solution of Leont'ev [10]. The present numerical results are plotted as continuous lines.

Figures 6-10 show that the Darcy number and Forchheimer term have similar and distinct effects on the flow field compared to the case of no-slip boundary condition. The similar aspects are:

- the decrease in $D a$ increases the values of the surface vorticity for all $R e$ 


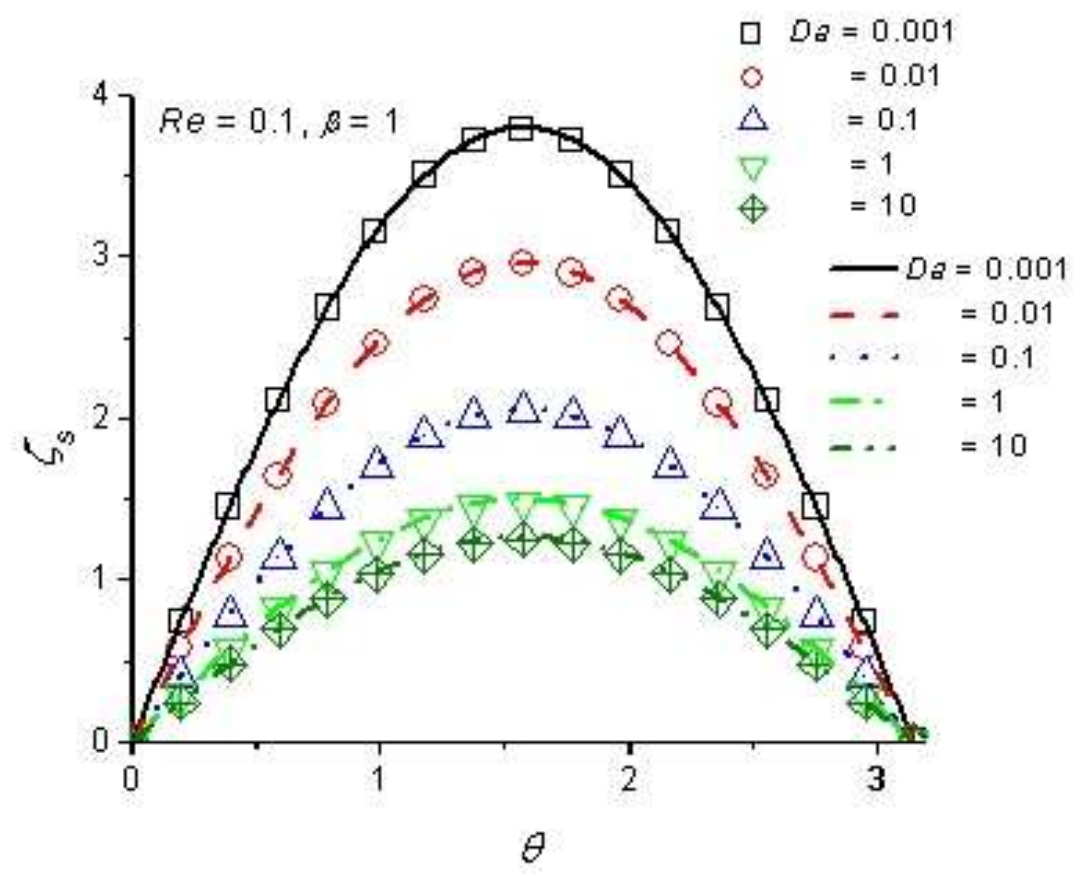

Figure 6: The influence of the $D a$ number on the surface vorticity for $R e=$ $0.1, \beta=1$ and $\varepsilon=0.9$.

number values;

- for $R e=0.1$ the influence of the Forchheimer term on the surface vorticity is negligible.

The distinct aspects are:

- for $R e=10$ the influence of the Forchheimer term on the surface vorticity is negligible for very small $D a$ values; for $D a=0.001$ and $R e=10$ the agreement between the present numerical results and the analytical solution of Leont'ev [10] is very good;

- the case $R e=1$ can be viewed as an intermediary one between the cases $R e=0.1$ and $R e=10$.

It must be mentioned that the influence of the Darcy and Forchheimer terms on the tangential surface velocity is similar to that observed for surface vorticity; the decrease in $D a$ increases the values of the tangential surface velocity. 


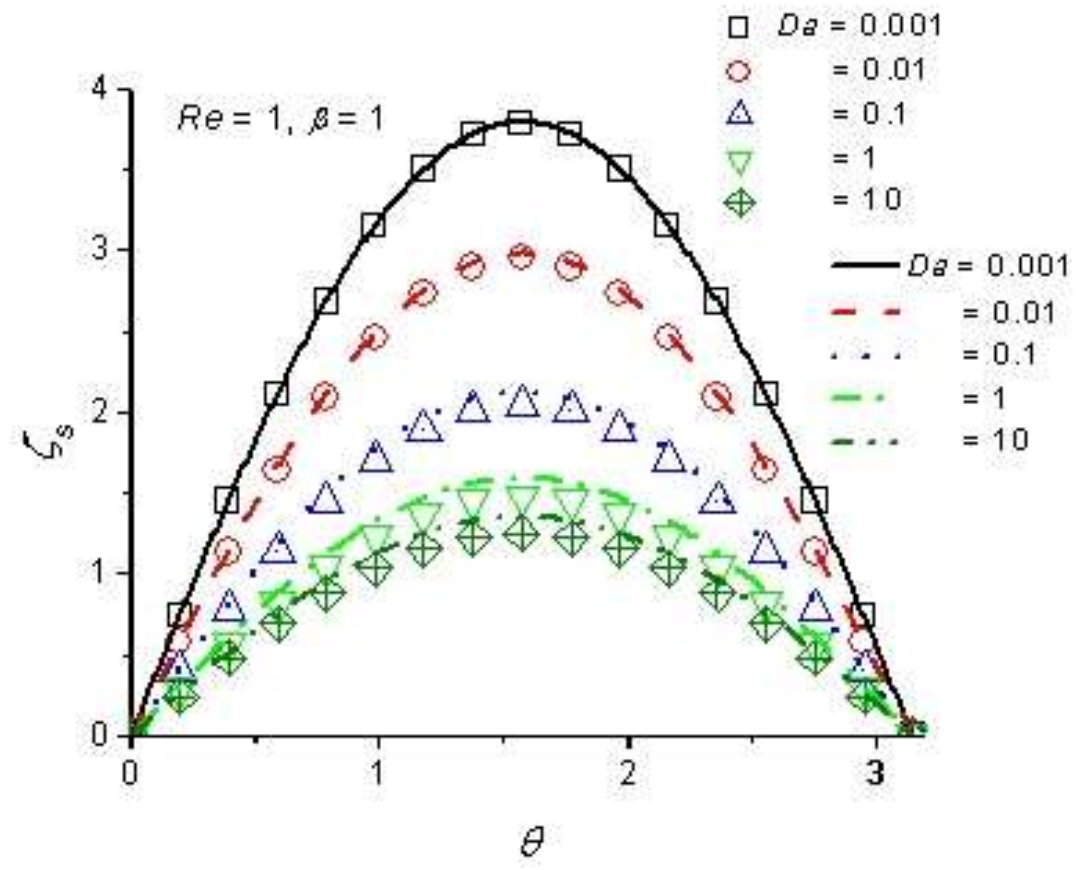

Figure 7: The influence of the $D a$ number on the surface vorticity for $R e=1$, $\beta=1$ and $\varepsilon=0.9$.

As was mentioned at the beginning of this section, the results discussed previously were computed for $\beta=1$. Numerical experiments were also made for $\beta=0.03125,0.0625,0.125,0.5$ and $\infty$. The numerical results obtained are similar to those calculated with $\beta=1$. However, as expected, the differences between the present results and the analytical solution from [10] increase when $\beta$ decreases. For example, for $R e=10$ and $D a=0.001$, the relative differences between the present results and the analytical solution of Leont'ev [10] are: (1) for $\beta=\infty$, approximately $1 \%$; (2) for $\beta=0.5$, smaller than $1 \%$; (3) for $\beta=0.125$, approximately $3 \%$; (4) for $\beta=0.0615$, approximately $5 \%$; (5) for $\beta=0.03125$, approximately $7 \%$.

Based on these numerical results, we may summarize that the effect of the Forchheimer term on the velocity profiles can be considered negligible for slip boundary conditions if:

- DaRe $<0.1$ for $R e \geq 1$ and $\beta \geq 0.1$; 
- $\operatorname{Re}<1$.

Under these conditions, the analytical solution of Leont'ev [10] can be used to calculate the velocity profiles.

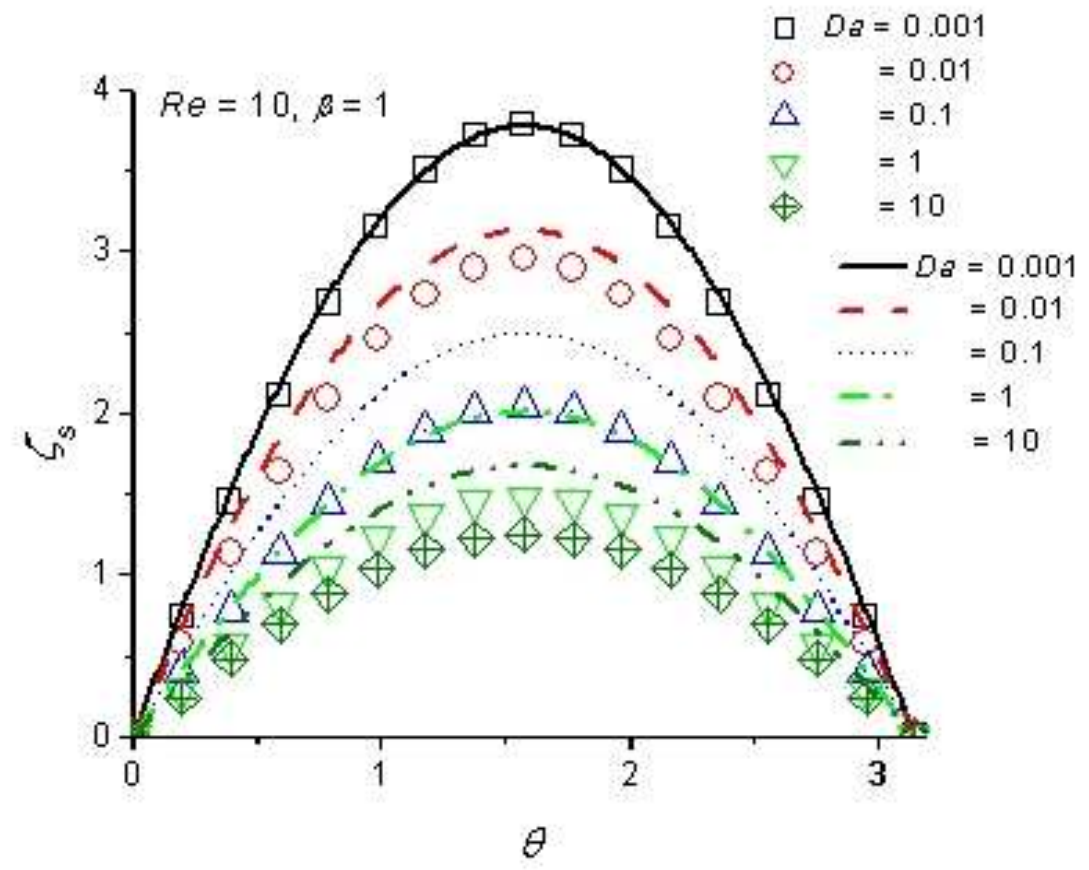

Figure 8: The influence of the $D a$ number on the surface vorticity for different Re numbers, $\beta=1$ and $\varepsilon=0.9$.

\section{Conclusions}

The objective pursued in this work is the numerical solution of the flow past an impermeable sphere embedded in a fluid saturated porous medium using the Brinkman-Forchheimer-Darcy model. The influence of the Da number and Forchheimer constant on the flow characteristics was investigated for sphere Re number in the range, $R e \leq 10$, and two types of boundary conditions on the surface of the sphere: slip and no - slip.

The numerical results presented in the previous section show that for no slip boundary conditions the influence of the Forchheimer term becomes sig- 


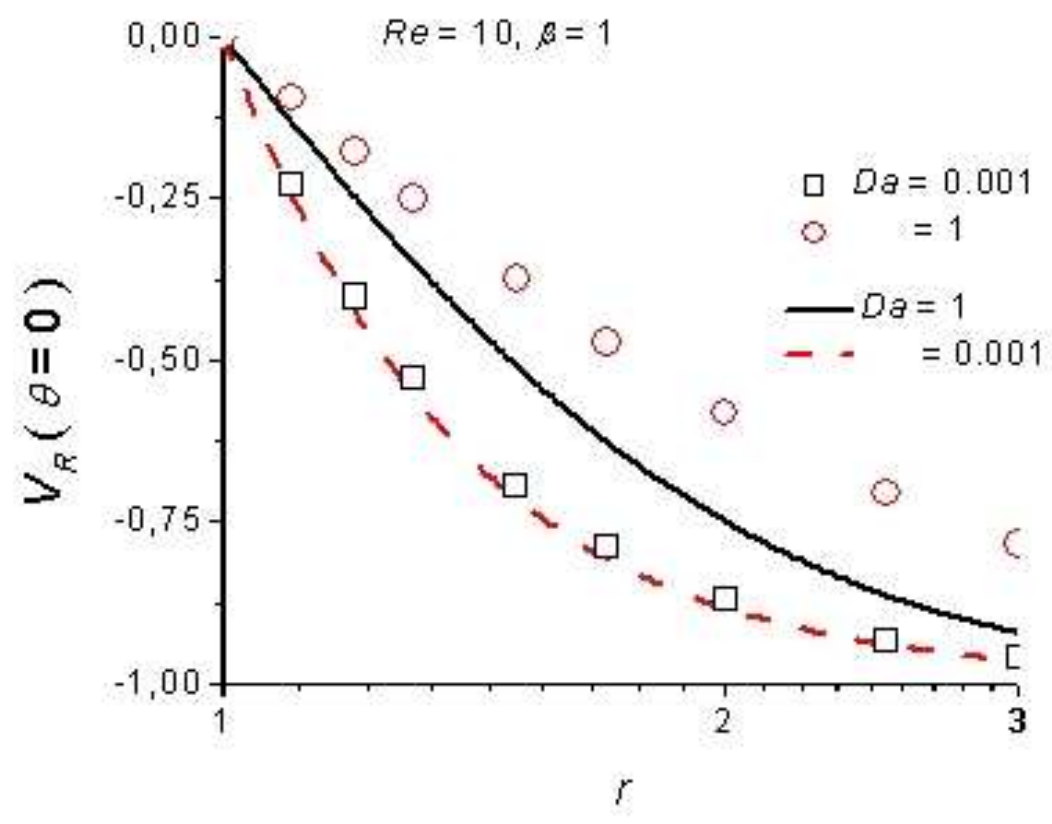

Figure 9: Radial velocity profiles for $\beta=1, D a=0.001,1, R e=10$ and $\varepsilon=0.9$.

nificant for $R e>1$. For $R e \leq 1$, the agreement between the present numerical results and the analytical solution of Wang [15] is very good. For slip boundary condition, the effect of the Forchheimer term on the velocity profiles depends on $\beta, D a$ and $R e$ values. It is negligible when DaRe $<0.1$ (Re $\geq 1$ and $\beta \geq 0.1$ ) and $R e<1$. When these conditions are satisfied, the analytical solution of Leont'ev [10] can be used.

\section{References}

[1] J. Bear, Y. Bachmat, Introduction to modeling of transport phenomena in porous media, Kluiwer Academic, Dordrecht (1990).

[2] B. Berman, Flow of a Newtonian fluid past an impervious sphere embedded in a porous medium, Indian J. Pure Appl. Math 27, 1249-1256 (1996). 


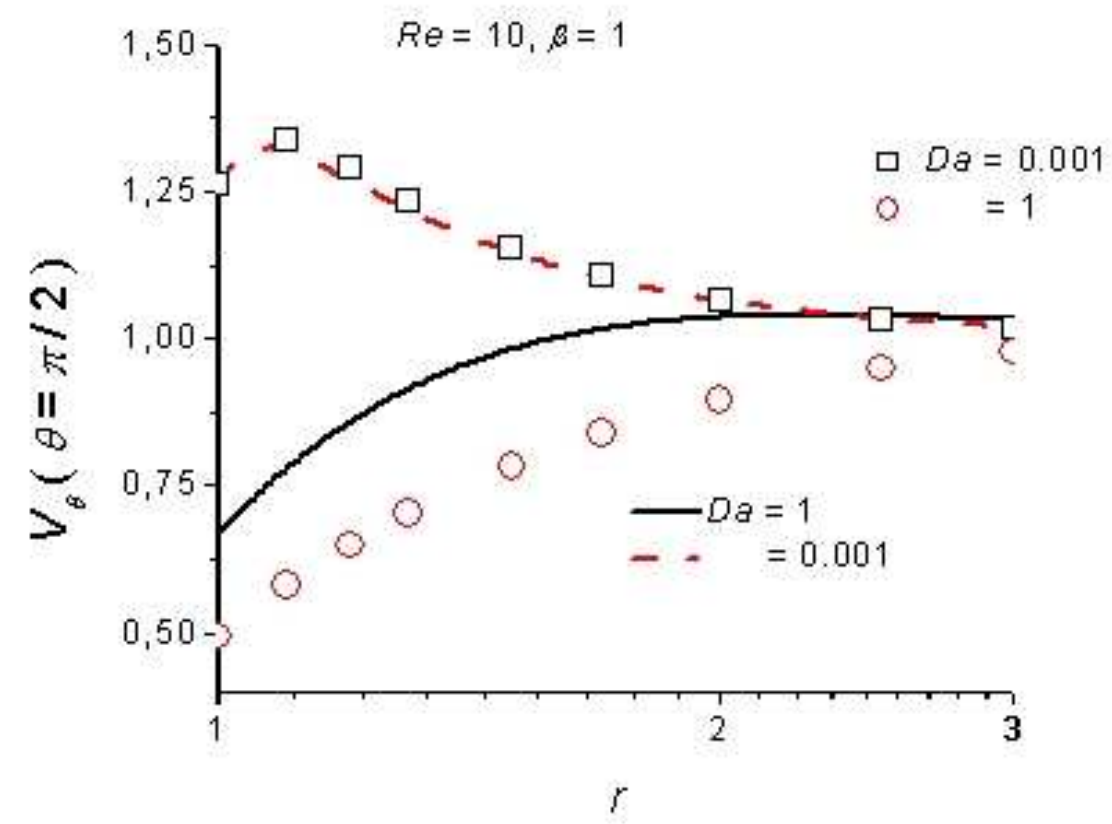

Figure 10: Tangential velocity profiles for $\beta=1, D a=0.001,1, R e=10$ and $\varepsilon=0.9$.

[3] A. Brandt, Multi-level adaptive solutions to boundary value problems, Math. Comput. 31, 333-390 (1977).

[4] H.C. Brinkman, A calculation of the viscous force exerted by a flowing fluid on a dense swarm of particles, Appl. Sci. Res. 1, 27-34 (1947).

[5] D.S. Charya, J.V.R. Murthy, Flow past an axisymmetric body embedded in a saturated porous medium, C.R. Mec. 330, 417-423 (2002).

[6] Z.-G. Feng, E.E. Michaelides, Unsteady mass transport from a sphere immersed in a porous medium at finite Peclet numbers, Int. J. Heat Mass Transfer 42, 535-546 (1999).

[7] R. Ganapathy, Creeping flow past a solid sphere in a porous medium, ZAMM 77, 871-875 (1997). 
[8] M. Kaviany, Principles of heat transfer in porous media, Second Edition, Springer, Berlin (1995).

[9] J.L. Lage, The fundamental theory of flow through permeable media: from Darcy to turbulence, In Transport Phenomena in Porous Media (eds. D.B. Ingham and I. Pop) Elsevier, Oxford, pp. 1-30 (1998).

[10] N.E. Leont'ev, Flow past a cylinder and a sphere in a porous medium within the framework of the Brinkman equation with the Navier boundary condition, Fluid Dynamics 49, 232-237 (2014).

[11] D.A. Nield, A. Bejan, Convection in Porous Media, Third Edition, Springer, New York (2006).

[12] I. Pop, D.B. Ingham, Flow past a sphere embedded in a porous medium based on the Brinkman model, Int. Commun. Heat Mass Transf. 23, 865874 (1996).

[13] L.A. Romero, Low or high Peclet number flow past a sphere in a saturated porous medium, SIAM J. Appl. Math. 54, 42-71 (1994).

[14] N. Rudraiah, I.S. Shivakumaran, D. Palaniappan, D.V. Chandrashekar, Flow past an impervious sphere embedded in a porous medium based on non-Darcy model, Adv. Fluid Mech. 2, 253-256 (2004).

[15] C.Y. Wang, Darcy Brinkman flow with solid inclusions, Chem. Eng. Commun. 197, 261-274 (2009).

Gheorghe Juncu,

Department of Chemical and Biochemical Engineering,

POLITEHNICA University Bucharest,

Polizu 1, 011061 Bucharest, Romania

Email: juncu@easynet.ro,juncugh@netscape.net 